\title{
Experimental Evaluation of Phase Noise Tolerance for Polarization-Insensitive Coherent Receiver
}

\author{
M. S. Erkılınç ${ }^{(1, *)}$, D. Lavery ${ }^{(1)}$, R. Maher ${ }^{(1)}$, B. C. Thomsen ${ }^{(1)}$, P. Bayvel ${ }^{(1)}$, R. I. Killey ${ }^{(1)}$, and \\ S. J. Savory ${ }^{(2)}$ \\ (1) Optical Networks Group, Department of Electronic \& Electrical Engineering, UCL (University College London), \\ London WC1E 7JE, UK \\ ${ }^{(2)}$ University of Cambridge, Department of Engineering, Electrical Engineering Division, $9 \mathrm{JJ}$ Thomson Avenue, \\ Cambridge, CB3 OFA, UK \\ ${ }^{(*)}$ m.erkilinc@ee.ucl.ac.uk
}

\begin{abstract}
The phase noise tolerance of a polarization-insensitive coherent receiver was experimentally assessed using Alamouti-coded OFDM signals. For a LO laser linewidth of $1 \mathrm{MHz}$, a sensitivity penalty of just $0.6 \mathrm{~dB}$ was observed for $10.7 \mathrm{~Gb} / \mathrm{s}$ OFDM-QPSK.
\end{abstract}

OCIS codes: (060.2330) Fiber optics communications, (060.1660) Coherent communications

\section{Introduction}

The increase in demand for bandwidth will inevitably require uncontended gigabit connections to the premises in optical access networks due to continuous growth of data intensive services such as high-definition video-on-demand and cloud computing. There is an imminent demand for optical network unit (ONU) data rates of several gigabits per second. Although time-division multiplexing (TDM) is currently used in such networks, it will be inherently limited in the number of subscribers supported, as TDM requires receivers with an electrical bandwidth many times greater than the per-user data rate [1]. Thus, coherent-enabled wavelength division multiplexed (WDM) access networks, in which each subscriber has a dedicated single wavelength, is a promising technology for future access networks [2,3]. Moreover, coherent reception offers superior receiver sensitivity providing a high split ratio ( $\geq 64$ users sharing the provider's operational costs), and resilience to chromatic dispersion offering a long reach (up to $100 \mathrm{~km}$ ) without any mid-span amplification [4].

On the other hand, the cost/size-per-bit constraints are particularly stringent in such systems. Therefore, despite the aforementioned advantages of coherent access networks, they are currently considered as impractical, mainly due to the high optical complexity of polarization- and phase-diverse intradyne (PPDI) coherent receivers. To reduce this receiver complexity, we have recently proposed a polarization-insensitive (PI) coherent receiver, implemented by applying polarization-time block (Alamouti) coding combined with heterodyne detection [5]. The optical complexity is significantly reduced whilst offering a high receiver sensitivity for a system operating at a net bit rate of $10 \mathrm{~Gb} / \mathrm{s}$ per wavelength [6]. In [5,6], an external cavity laser (ECL - $100 \mathrm{kHz}$ linewidth) was used as a local oscillator (LO) in our PI coherent receiver. It is desirable to further reduce the receiver optical complexity by using a high linewidth laser, which is a more conventional integrable device such as a distributed feedback (DFB) laser (a typical linewidth of approximately $1 \mathrm{MHz}$ ). However, Alamouti-coding has an orthogonal structure so that it is highly susceptible to phase noise, which introduces random rotations and reduces the block code orthogonality. The reduction in orthogonality depends on the laser linewidth which impacts directly on receiver sensitivity. Hence, it is crucial to mitigate the laser phase noise successfully so that high receiver sensitivities can be achieved.

In this work, the impact of laser phase noise on the receiver sensitivity of Alamouti-coded OFDM (AC-OFDM) QPSK and 16-QAM signals (operating at net bit rates of 10 and $20 \mathrm{~Gb} / \mathrm{s}$, respectively) was assessed using the PI coherent receiver. An RF-pilot aided phase noise mitigation scheme was utilized for the AC-OFDM signal formats. This scheme has been previously studied for linewidths of up to $400 \mathrm{kHz}$ and shown to exhibit a considerably higher linewidth tolerance compared to the common phase error based mitigation scheme [7]. Herein, the laser linewidth values were varied from $0.1 \mathrm{to} 10 \mathrm{MHz}$ using a phase noise emulator that was used to change the linewidth of the LO laser (from $0.1 \mathrm{MHz}$ to $10 \mathrm{MHz}$ ). Subsequently, a DFB laser with a $1 \mathrm{MHz}$ linewidth was used as the LO laser to validate the receiver sensitivity measurements.

\section{Experimental Configuration}

The experimental setup for the laser phase noise tolerance study of the Alamouti-coded OFDM signal, received using the PI coherent receiver, is shown in Fig. 1. In the transmitter, $10.7 \mathrm{~Gb} / \mathrm{s}$ AC-OFDM QPSK and $21.4 \mathrm{~Gb} / \mathrm{s}$ AC-OFDM 16-QAM signals, including the 7\% overhead reserved for the hard-decision forward error correction (HD-FEC), were generated using a dual-polarization (DP) in-phase quadrature (IQ) modulator. An ECL with a linewidth of $100 \mathrm{kHz}$ was used as a source laser 
and the modulator was driven by four driving signals, implemented offline in the transmitter digital signal processing (TxDSP) and uploaded to the memory of two arbitrary waveform generators (ArbWGs), operating at $12 \mathrm{GSa} / \mathrm{s}$. A 512-point fast Fourier transform (FFT), yielding a carrier spacing of approximately $235 \mathrm{MHz}$, was used and 316 subcarriers were modulated with data (QPSK and 16-QAM), achieving pre-FEC bit rates of 10.7 and $21.4 \mathrm{~Gb} / \mathrm{s}$, respectively. The QPSK and 16-QAM waveforms were clipped to obtain peak-to-average power ratios of 7 and $9 \mathrm{~dB}$, respectively. Subsequently, an optical carrier (with a carrier-to-signal power ratio (CSPR) of -11 dB) was added on both polarizations by slightly detuning the modulator away from its null point. The carrier, which appears as an RF-pilot tone following the heterodyne detection using the PI coherent receiver, was utilized in the phase noise $(\mathrm{PN})$ mitigation stage at the receiver digital signal processing (Rx-DSP). Note that the reason for inserting the carrier on both polarizations is to avoid power fading due to polarization rotations. Depending on the total linewidth of the system, the minimum number of null subcarriers around the DC frequency was determined to maximize the spectral efficiency, as shown in the inset (a) of Fig. 1. This is further discussed in the next section. The detailed discussion regarding the DSP blocks to generate the AC-OFDM frame is included in [6].

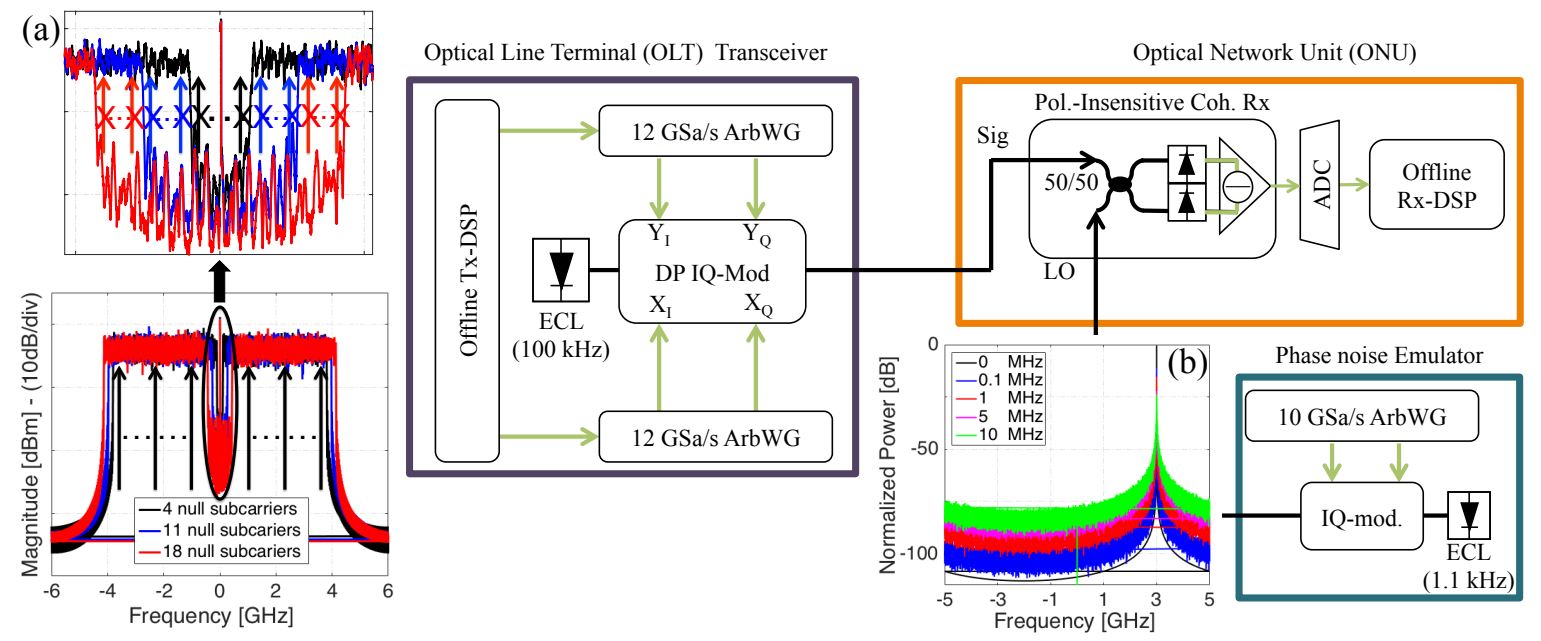

Fig. 1. Experimental setup. Insets: (a) Electrical spectrum uploaded to ArbWG memory (zoomed around the DC frequency to illustrate the null subcarriers). (b) Electrical spectra uploaded to the ArbWG memory to emulate the laser phase noise.

At the receiver, the PN emulator, proposed in [8], was used to manipulate the linewidth of the LO laser. A subcarrier at a frequency of $3 \mathrm{GHz}$ with an emulated PN (varying the linewidth from 0.1 to $10 \mathrm{MHz}$ ) was generated offline in Matlab, and subsequently, the waveforms were uploaded to the ArbWG (operating at $10 \mathrm{GSa} / \mathrm{s}$ ) memory to be used as the driving signals, as shown in the inset (b) of Fig. 1. The IQ modulator, seeded by a narrow linewidth ECL whose linewidth was negligible compared that generated by the PN emulator, was biased at its null point to generate an optical carrier ( $3 \mathrm{GHz}$ away from the narrow linewidth ECL) with the targeted PN. Following this, it was coherently mixed with the transmitted (AC-OFDM) signal, and detected by using the PI coherent receiver, consisting of a single balanced photodiode and an ADC. Following the detection, the RF-pilot tone aided scheme was performed to mitigate the PN, as proposed in [9]. The tone was first filtered using a $5^{\text {th }}$-order Butterworth LPF. Following this, the filtered signal was conjugated and multiplied with the received signal to mitigate the laser PN. Note that the filter bandwidth was adjusted to achieve the optimum performance at each linewidth value. The details of the offline Rx-DSP blocks for the AC-OFDM signal demodulation are reported in [6]. The receiver sensitivity performance of the AC-OFDM signals was measured by bit error rate (BER) estimation.

\section{Experimental Results and Discussions}

Initially, the minimum number of null subcarriers required around the DC frequency was determined for each linewidth value, as shown in Fig. 2(a). It is found to be only 8 subcarriers (causing a $2.5 \%$ bandwidth overhead) for $0.1 \mathrm{MHz}$ whereas it gradually increases to 16 and 36 subcarriers (introducing 5\% and $11 \%$ bandwidth overheads) for 1 and $10 \mathrm{MHz}$ linewidths, respectively. In addition to this, it is important to optimize the LPF bandwidth used for filtering the RF-pilot tone. The system performance is limited by the residual laser phase noise (after PN mitigation) if the LPF bandwidth is not sufficiently high to capture the PN information whereas the electrical noise limits the performance when the bandwidth is excessively high. The optimum LPF bandwidth is found to be $5 \mathrm{MHz}$ for $0.1 \mathrm{MHz}$ linewidth whereas it increases to 25 and $70 \mathrm{MHz}$ at 1 and $10 \mathrm{MHz}$ linewidths, respectively. Note that the optimum CSPR per polarization was found to be $-11 \mathrm{~dB}$ for all the linewidths. Following this, although the minimum number of null subcarriers required for the linewidth values $<10 \mathrm{MHz}$ was less than 36 subcarriers, it was kept the same, causing a $0.8 \mathrm{GHz}$ bandwidth overhead to operate at the same net spectral 


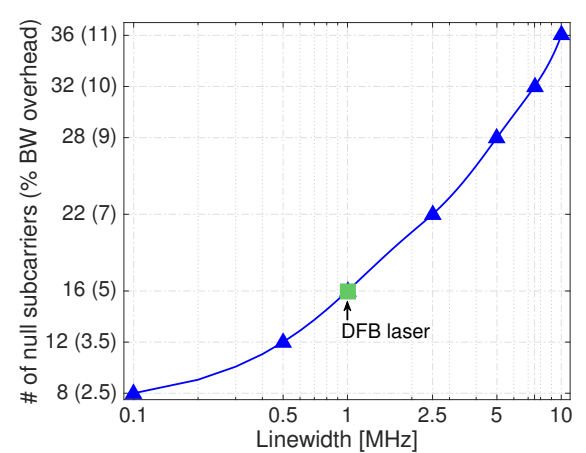

(a)

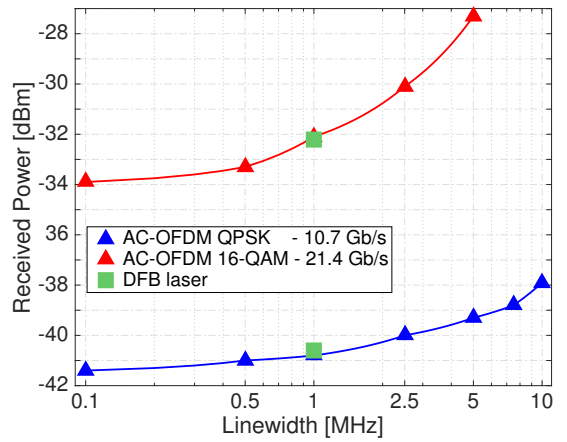

(b)

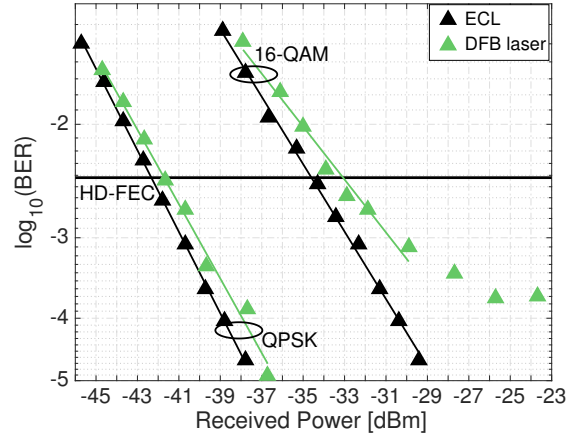

(c)

Fig. 2. (a) The minimum number of null subcarriers required for the PN mitigation to achieve the optimum receiver sensitivity, (b) the receiver sensitivities with respect to the LO laser linewidth using the PN emulator, and (c) BER vs received power using the ECL and DFB laser (0.1 and $1 \mathrm{MHz}$ linewidth), matching well with the PN emulation results shown in (b).

efficiency in all cases.

The receiver sensitivities, achieving the HD-FEC threshold (assumed to be $4 \times 10^{-3}$ ), with respect to the laser linewidth for the AC-OFDM QAM signals using the PN emulator are shown in Fig. 2(b). The LO power was set to $12 \mathrm{dBm}$ for all the sensitivity measurements. The required receiver sensitivities at the HD-FEC threshold gradually increases when the linewidth of the LO laser increases, as can be observed in Fig. 2(b). As expected, the increase in sensitivity penalty for the 16-QAM signal is significantly higher than the QPSK signal, with the system failing to achieve the HD-FEC threshold at the very high linewidths $(7.5$ and $10 \mathrm{MHz}$ ). At a linewidth of $0.1 \mathrm{MHz}$, the receiver sensitivity for the $10.7 \mathrm{~Gb} / \mathrm{s}$ AC-OFDM QPSK signal was measured to be $-41.5 \mathrm{dBm}$. When the modulation scheme was changed from QPSK to 16-QAM at the same symbol rate, a total sensitivity penalty of $7.5 \mathrm{~dB}(6 \mathrm{~dB}$ penalty due to the decrease in minimum Euclidean symbol spacing, $0.8 \mathrm{~dB}$ due to the limited resolution of DACs/ADC and $0.7 \mathrm{~dB}$ after the PN mitigation) was observed since 16-QAM signals have lower resilience to laser $\mathrm{PN}$ and nonlinear distortion caused by clipping. When the linewidth was broadened to $1 \mathrm{MHz}$, the penalty was increased to $9 \mathrm{~dB}$ due to the higher susceptibility of the 16-QAM signal to laser PN, as can be seen in Fig. 2(b).

Following the PN emulation, an ECL and a DFB laser with linewidths of 0.1 and $1 \mathrm{MHz}$ were used to verify our phase noise tolerance study for the AC-OFDM signal and PI coherent receiver. The BER curve with respect to the received power is shown in Fig. 2(c). When the DFB laser was used as a LO laser, the received power values achieving the HD-FEC threshold for the AC-OFDM QPSK and 16-QAM signals were found to be -40.9 and $-32 \mathrm{dBm}$, respectively. Note that the DFB points agree perfectly with the emulated PN providing good evidence for using the emulated phase noise approach, as can be observed in Fig. 2(b).

\section{Conclusions}

We experimentally investigated the laser phase noise resilience of our recently proposed polarization insensitive coherent receiver, enabled by Alamouti coding and heterodyne reception. The receiver sensitivity performance of pre-FEC 10.7 and $21.4 \mathrm{~Gb} / \mathrm{s}$ AC-OFDM QPSK and 16-QAM was assessed by varying the linewidth of the LO laser using a phase noise emulator. Following this, a DFB laser with a linewidth of $1 \mathrm{MHz}$ was employed to verify the emulation. Our study indicates that the Alamouti-coded OFDM signals, which are highly phase sensitive, can be successfully recovered with linewidths of up to $5 \mathrm{MHz}$. Sensitivity penalties of 0.6 and $2 \mathrm{~dB}$ were measured for Alamouti-coded OFDM QPSK and 16-QAM signal formats, respectively when a DFB laser with a linewidth of $1 \mathrm{MHz}$ was used as the $\mathrm{LO}$ laser in the receiver.

\section{References}

1. ITU-T recommendation G.989.1, "40-Gigabit-capable passive optical networks (NG-PON2): General requirements" (2013).

2. H. Rohde et al., "Coherent ultra dense WDM technology for next generation optical metro and access networks," JLT 32, 2041-2052 (2014).

3. A. Shahpari et al., "Coherent ultra dense wavelength division multiplexing passive optical networks," OFT 26, 100-107 (2015).

4. D. Lavery et al., "Bidirectional $10 \mathrm{Gbit/s}$ long-reach WDM-PON using digital coherent receivers", in Proc. OFC'11, paper OTuB4 (2011).

5. M.S. Erkılınç et al., "Polarization-insensitive single balanced photodiode coherent receiver for passive optical networks," in Proc. ECOC'15, paper Th.1.3.3 (2015).

6. M.S. Erkılınç et al., "Polarization-insensitive single-balanced photodiode coherent receiver for long-reach WDM-PONs," JLT 34, 2034-2041 (2016).

7. S. Randel et al., "Analysis of RF-pilot-based phase noise compensation for coherent optical OFDM systems," IEEE PTL 22, 1288-1290 (2010).

8. Z. Zan and A.J. Lowery, "Experimental demonstration of a flexible and stable semiconductor laser linewidth emulator," Opt. Express 18, 1388013885 (2010).

9. S.L. Jansen et. al., "Coherent optical 25.8-Gb/s OFDM transmission over 4160-km SSMF,” JLT 26, 6-15 (2008). 